\title{
U.S. Hydropower Resource Assessment for
}

North Dakota

James E. Francfort

Published December 1993

\author{
Idaho National Engineering Laboratory \\ EG\&G Idaho, Inc. \\ Idaho Falls, Idaho 83415
}

Prepared for the

U.S. Department of Energy

Assistant Secretary for Energy Efficiency and Renewable Energy Idaho Operations Office

Under DOE Contract DE-AC07-76ID01570

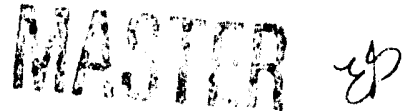




\begin{abstract}
The Department of Energy is developing an estimate of the hydropower development potential in this country. The Hydropower Evaluation Software $(f \quad E S)$ is a computer model that was developed by the Idaho National Engineering Laboratory for this purpose. The HES measures the potential hydropower resources available in the United States, using uniform criteria for measurement. The software was developed and tested using hydropower information and data provided by the Southwestern Power Administration. It is a dBASE menu-driven software application that allows the personal computer user to assign environmental attributes to potential hydropower sites, calculate development suitability factors for each site based on the environmental attributes present, and generate reports based on these suitability factors. This report details the resource assessment results for the state of North Dakota.
\end{abstract}




\section{CONTENTS}

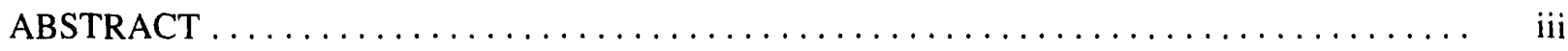

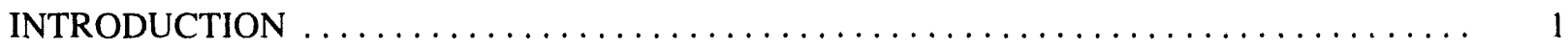

Model Development $\ldots \ldots \ldots \ldots \ldots \ldots \ldots \ldots \ldots \ldots \ldots \ldots \ldots \ldots \ldots \ldots \ldots \ldots \ldots \ldots \ldots$

Model Goal $\ldots \ldots \ldots \ldots \ldots \ldots \ldots \ldots \ldots \ldots \ldots \ldots \ldots \ldots \ldots \ldots \ldots \ldots \ldots \ldots \ldots \ldots$

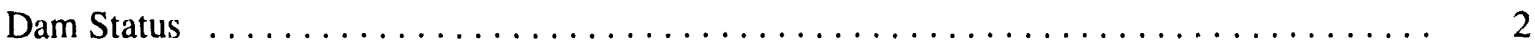

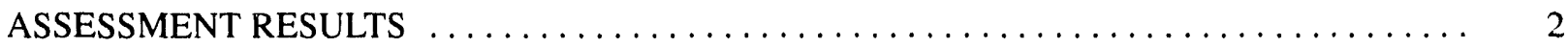

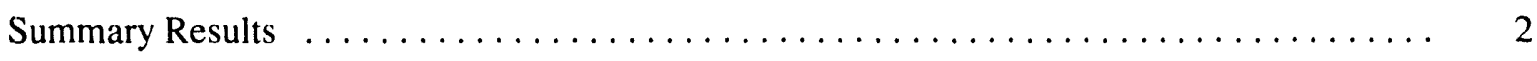

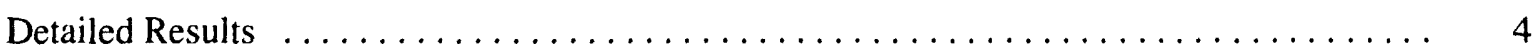

OBTAINING INDIVIDUAL STATE INFORMATION $\ldots \ldots \ldots \ldots \ldots \ldots \ldots \ldots \ldots \ldots$

ADDITIONAL HYDROPOWER EVALUATION SOFTWARE INFORMATION $\ldots \ldots \ldots \ldots \ldots .6$

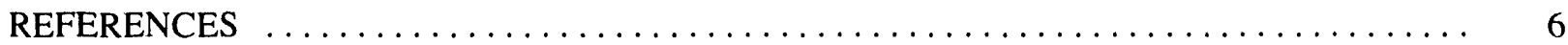

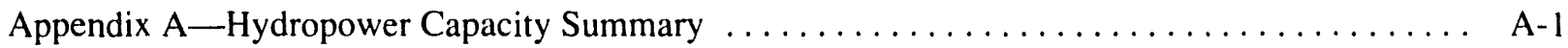

Appendix B-Hydropower Resource Assessment by River Basin $\ldots \ldots \ldots \ldots \ldots \ldots \ldots \ldots$ B-1

Appendix C - North Dakota (ND) Sites $\ldots \ldots \ldots \ldots \ldots \ldots \ldots \ldots \ldots \ldots \ldots \ldots \ldots \ldots \ldots \ldots \ldots \ldots$

Appendix D-Resource Database Listing $\ldots \ldots \ldots \ldots \ldots \ldots \ldots \ldots \ldots \ldots \ldots \ldots \ldots \ldots \ldots \ldots \ldots \ldots \ldots$

\section{FIGURES}

1. Number of sites with various capacity potentials $\ldots \ldots \ldots \ldots \ldots \ldots \ldots \ldots \ldots \ldots$

2. The Hydropower Evaluation Software identified potential hydropower capacity

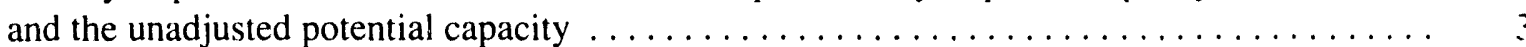

3. The number of sites with potential hydropower capacity and the total

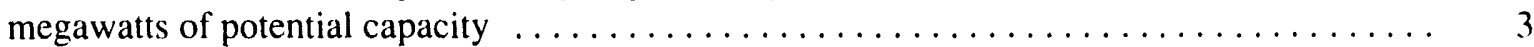

4. Number of sites with potential hydropower capacity in the North Dakota river basins ...... 4

5. Potential hydropower capacity in the North Dakota river basins $\ldots \ldots \ldots \ldots \ldots \ldots \ldots \ldots$ 


\section{U.S. Hydropower Resource Assessment for North Dakota}

\section{INTRODUCTION}

In June 1989, the U.S. Department of Energy initiated the development of a National Energy Strategy to identify the energy resources available to support the expanding demand for energy in the United States. Public hearings conducted as part of the strategy development process indicated that potential hydropower resources were not well defined. As a result, the Department of Energy established an interagency Hydropower Resource Assessment Team to ascertain the hydropower potential. In connection with these efforts by the Department of Energy, the Idaho National Engineering Laboratory designed the Hydropower Evaluation Software (HES), which has been used to perform a resource assessment of the undeveloped hydropower potential in North Dakota (as well as several other states). This report presents the results of the hydropower resource assessment for the state of North Dakota. Pumped storage hydropower potential is not included.

The HES was developed as a tool to measure hydropower potential by regional power marketing administrations and state energy agencies, because they were the most likely to have and need accurate hydropower information. The HES was not intended to provide precise development factors for individual sites, but to provide regional or state capacity totals. Because the software was developed as a generic measurement tool encompassing national issues, regional and state totals must be considered judiciously; various local issues may skew hydropower potential totals. The information for the resource assessment was compiled from the Federal Energy Regulatory Commission's Hydropower Resource Assessment database and several other sources. Refer to DOE/ID-10338, the User's Manual (Francfort, Matthews, Rinehart, 1991) for the specifics of the software and to DOE/ID-10430, the Status Report (Francfort, Moore, Rinehart, 1993) for an overview of all resource assessment activities to date.

\section{Model Development}

Hydropower Evaluation Software, both a probability-factor computer model and a data base, is a dBASE, menu-driven software application that is intended to be user-friendly. Computer screens and report generation capabilities were developed to meet the needs of users nationwide. The software uses environmental attribute data to generate an overall project environmental suitability factor (PESF) between 0.1 and 0.9 , where 0.9 indicates the highest likelihood of development and 0.1 indicates the lowest likelihood of development. Suitability factors depend on environmental attributes of a potential site. They reflect the considerations that (a) environmental concerns can make a potential site unacceptable. prohibiting its development (for a suitability factor of 0.1 ), or (b) absence of environmental concern can have little or no effect on the likelihood of site development (for a suitability factor of 0.9 ). A combination of attributes results in a lower suitability factor because multiple environmental considerations reduce the likelihood that a site may be developed to its physical potential.

\section{Model Goal}

The goal of the HES is to assemble an accurate resource database of all potential hydropower sites in the United States for use as a planning tool to determine the viable national hydropower potential. Potential hydropower is not limited to the development of new sites; it also includes the development of additional hydropower at sites that currently have hydropower but are not developed to their full potential. This hydropower potential is a source of nonpolluting, renewable energy available to meet the growing power needs of the United States. The HES should make 
this goal attainable and help assure a set of uniform criteria for national assessment.

\section{Dam Status}

The effects of environmental attributes vary by dam status. The dam status classifications follow the Federal Energy Regulatory Commission (FERC) standard, which is

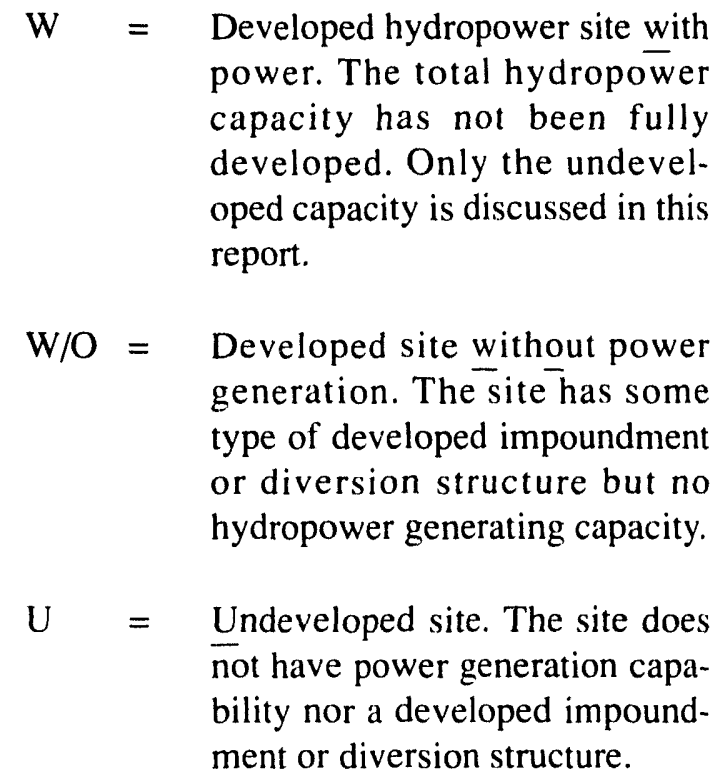

\section{ASSESSMENT RESULTS}

\section{Summary Results}

A total of 14 sites have been identified and assessed for their hydropower potential. Information as to the potential megawatts of capacity for 2 of the sites was not available; however, these sites have been identified as having hydropower potential and are included in the group of 14. The Hydropower Evaluation Software results for site capacities range from 1 kilowatt to 43 megawatts. Most of the sites have potential capacities of under 1 megawatt (Figure 1).

The unadjusted hydropower potential for North Dakota was identified as being 100 megawatts. The Hydropower Evaluation Software results lower this estimate $50 \%$ to 50 megawatts. The greatest reduction in undeveloped potential occurs at sites with some hydropower already installed. These sites have a Hydropower Evaluation Software estimated capacity of 43 megawatts, a $50 \%$ reduction in capacity (Figure 2 ). The number of sites does not change, only the identified capacity is reassessed (Figure 3 ).

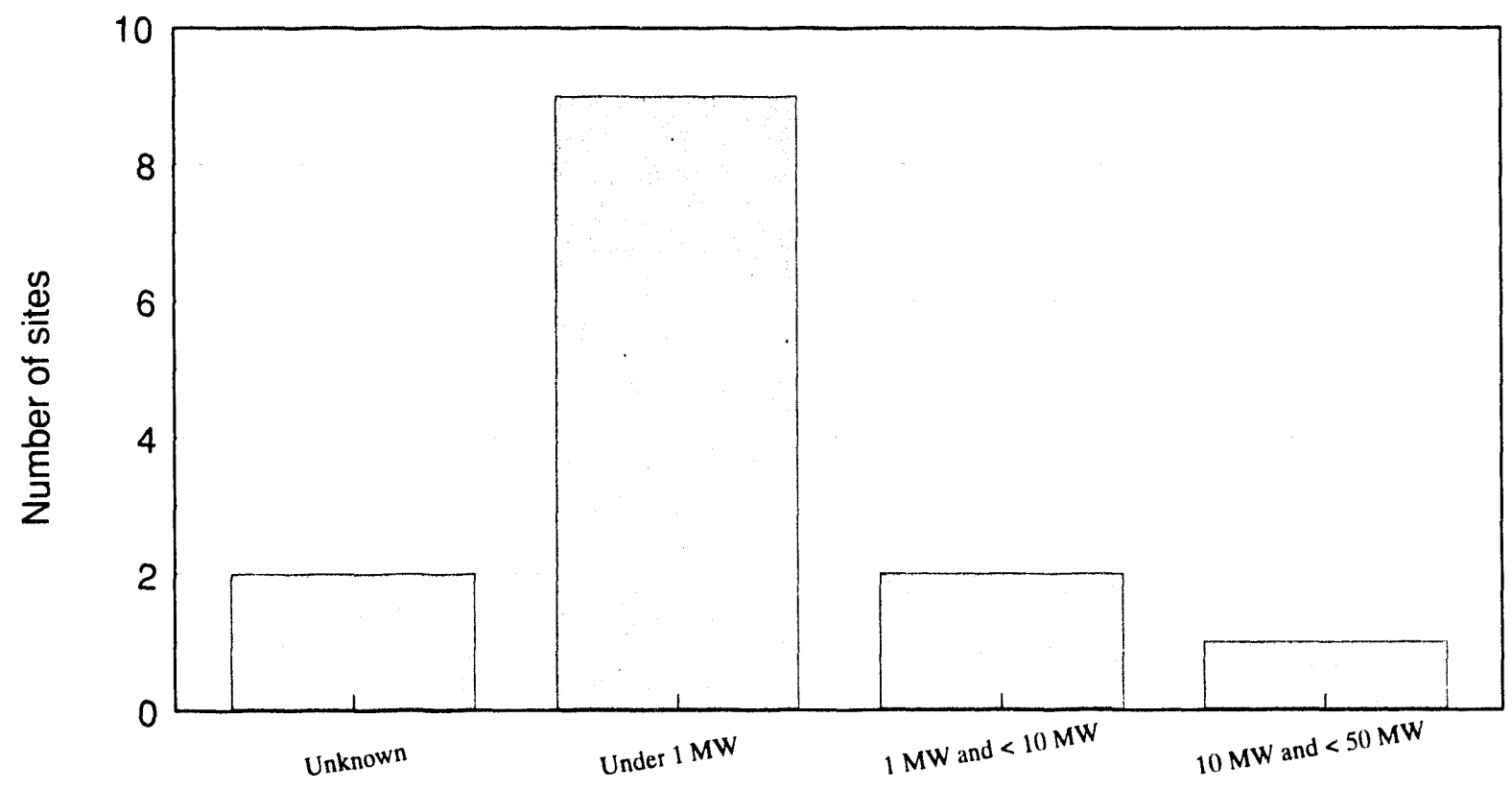

Figure 1. Number of sites with various capacity potentials. The unknown category is for sites that have hydropower potential but the size of the potential is undefined. 


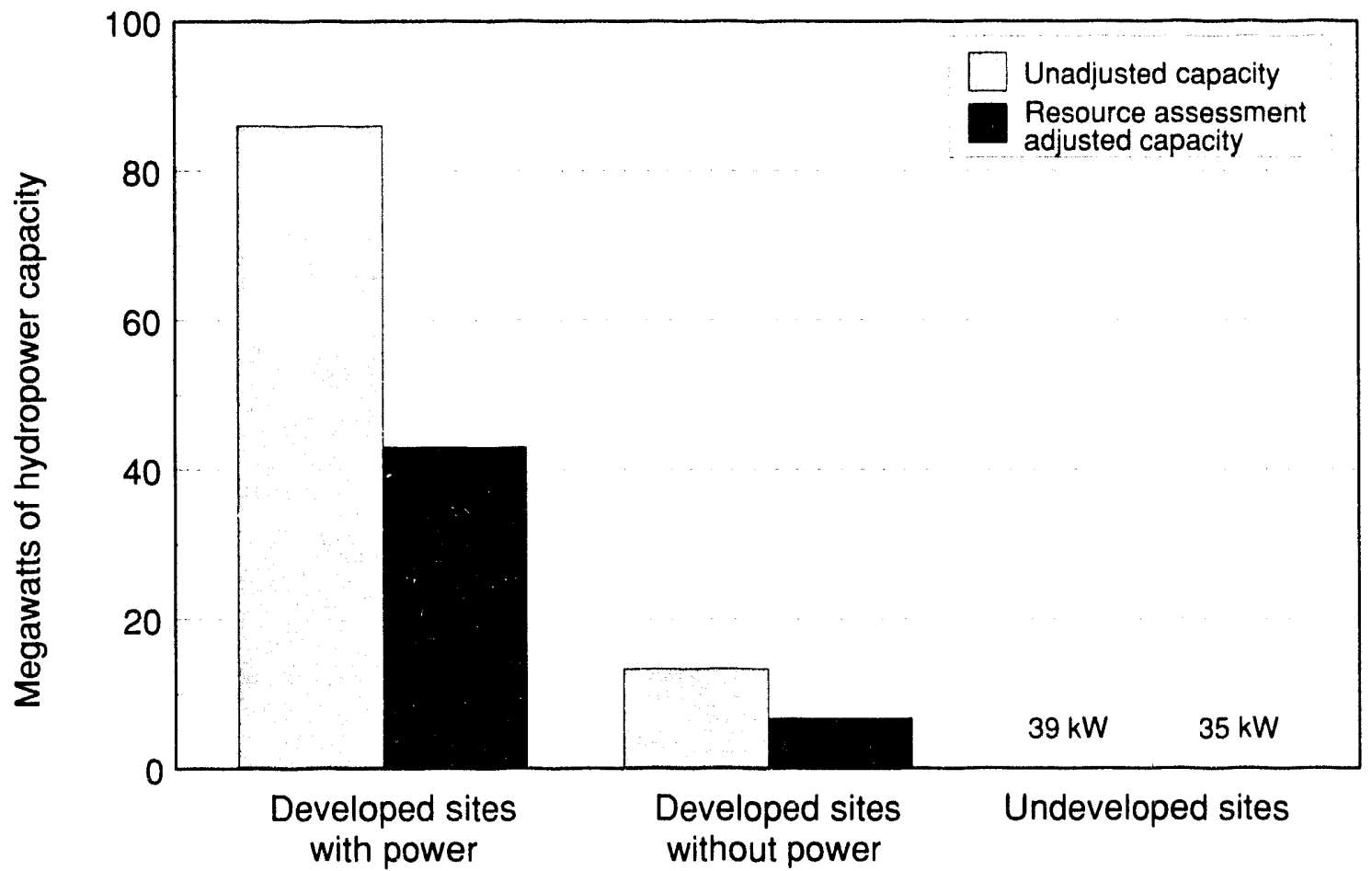

Figure 2. The Hydropower Evaluation Software identified potential hydropower capacity and the unadjusted potential capacity.

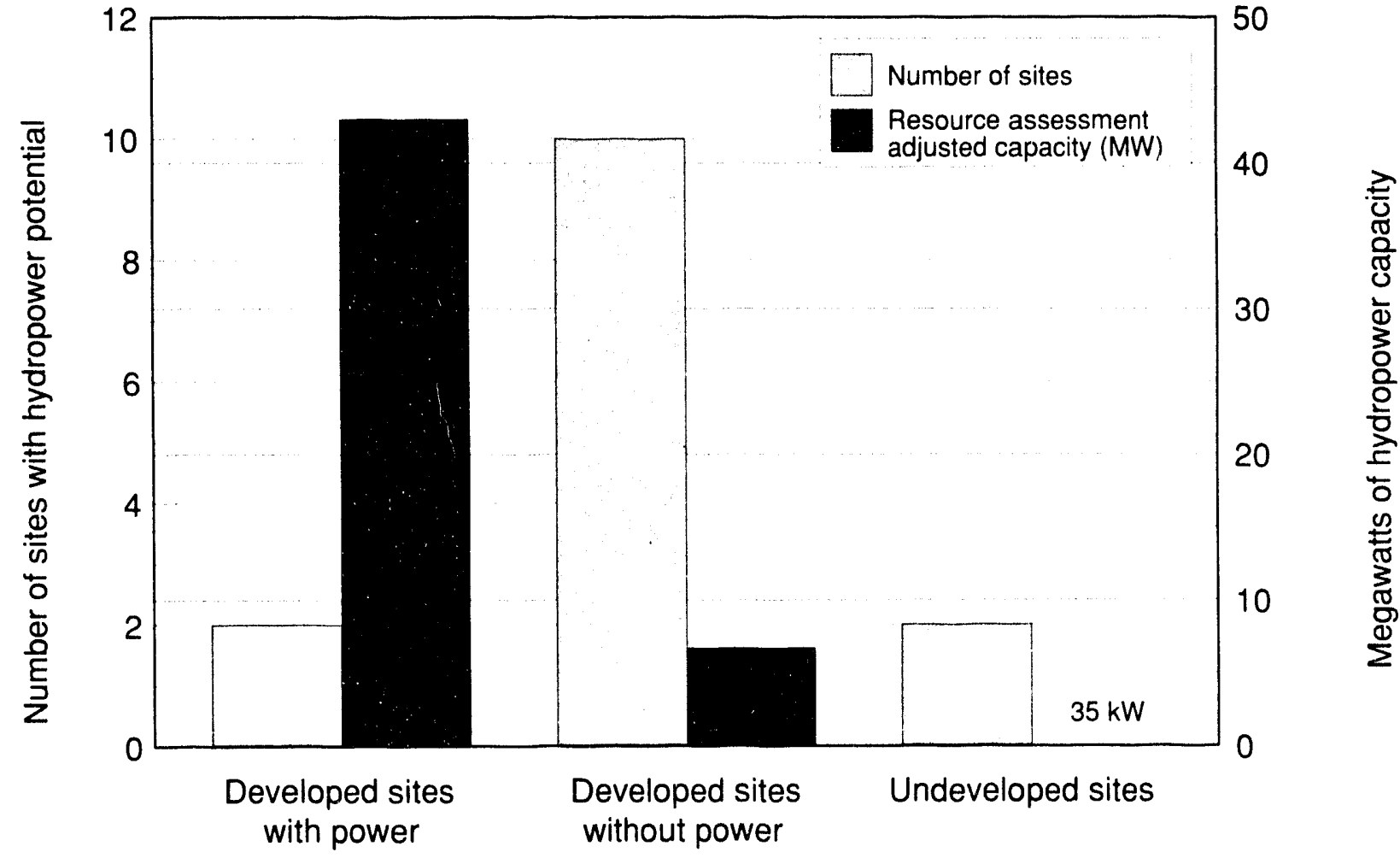

Figure 3. The number of sites with potential hydropower capacity and the total megawatts of potential capacity. 
The 14 identified sites are located within 10 river basins. The number of sites per river basin ranges from 1 site in several river basins to 3 sites in the Red River of the North River Basin (Figure 4). The Missouri River Basin has the most undeveloped potential hydropower capacity of the North Dakota river basins (Figure 5).

\section{Detailed Results}

The appendices contain, in the form of Hydropower Evaluation Software generated reports, detailed information of the potential hydropower capacity in North Dakota. The appendices contain the following information:

Appendix A The hydropower capacity summary for North Dakota categorizes sites by dam status. The number of sites, unadjusted capacity, and Hydropower Evaluation Software adjusted capacity are provided based on the dam status.

Appendix B The hydropower resource assessment by river basin includes the FERC project number, project name, stream name, dam status, unadjusted capacity, and Hydropower Evaluation Software adjusted capacity for each of the individual sites. Subtotals are provided for each river basin.

Appendix C This is a listing of the project numbers, plant name, stream name, if a site is Federally owned, unadjusted capacity, and Hydropower Evaluation Software adjusted capacity. The sites are grouped by dam status.

Appendix D This section contains a resource database listing for each of the 14 sites in North Dakota. Information includes plant, stream, state, county, river basin and owner names; project number; name plate and Hydropower Evaluation Software adjusted capacity ratings; the unit and plant types, dam status; latitude; longitude; and the environmental factors that the Hydropower Evaluation Software uses to determine the project environmental suitability factor.

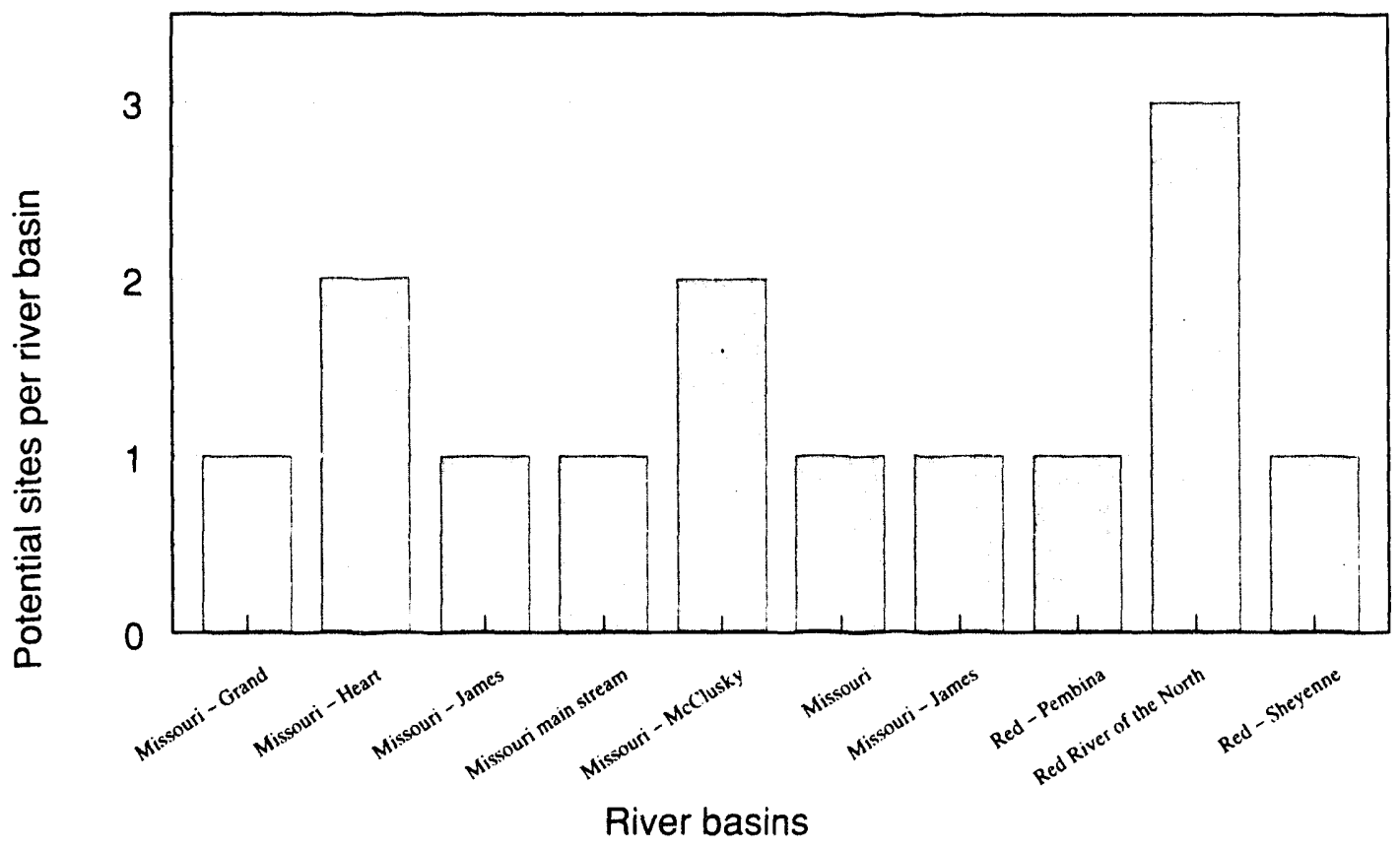

Figure 4. Number of sites with potential hydropower capacity in the North Dakota river basins. 


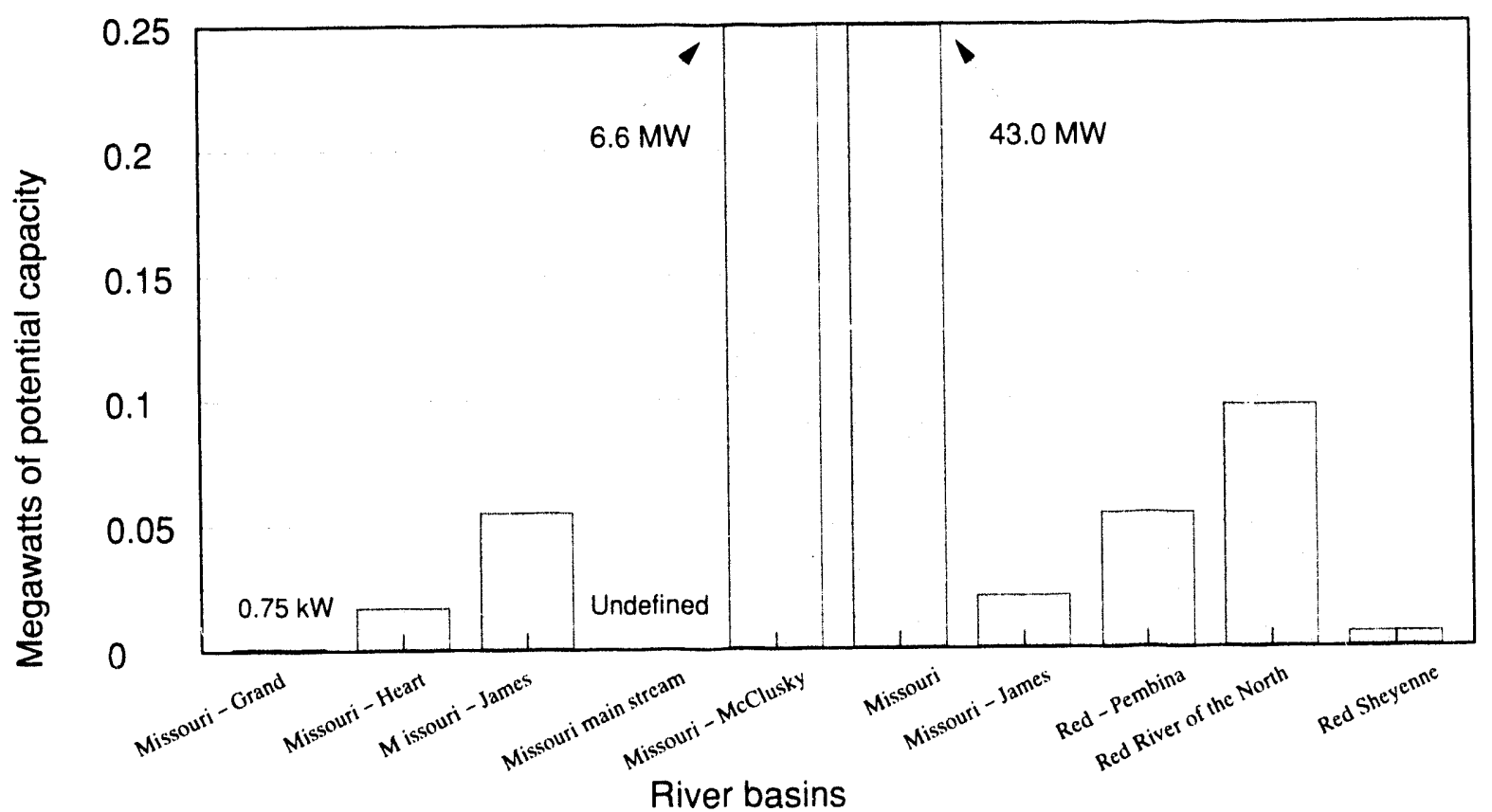

Figure 5. Potential hydropower capacity in the North Dakota river basins.

\section{OBTAINING INDIVIDUAL STATE INFORMATION}

Additional copies of the hydropower resource assessment results for individual states are available and can be obtained by writing or calling the National Technical Information Service (NTIS).

Telephone Orders - (703) 487-4650. NTIS sales desk and customer services are available between 8:30 a.m. and 5:00 p.m., Eastern Standard Time.

Fax - (703) 321-8547. Customers may fax their orders to NTIS. These orders may be charged to a NTIS deposit account, American Express, VISA, or MasterCard.

Mail Orders - Mail orders should be sent to National Technical Information Service, Document Sales, 5285 Port Royal Road, Springfield, VA 22161. Call the sales desk for prices before placing an order.
Method of Payment - Customers may pay for reports (and other NTIS products and services) by (a) credit card (American Express, Visa, or MasterCard); (b) check or money order on a United States bank payable to NTIS; (c) a NTIS deposit account; or (d) by asking to be billed (add $\$ 7.50$ per order, United States, Canada, and Mexico, only).

Handling Fee - A $\$ 3.00$ handling fee per total order applies to orders from the United States, Canada, and Mexico. Handling charges do not apply to rush order service or pick-up orders.

Postage and Shipping - Orders are shipped first class mail, or equivalent, to addresses in the United States, Canada, and Mexico.

Order Turnaround Time - Orders for technical reports generally are shipped within 2 to 8 days of receipt. For faster service, NTIS offers rush order service.

Rush Order Service - Call 1-800-533-NTIS. In Virginia, Canada, and Mexico call (703) 487-4700. For NTIS rush order service add 
$\$ 15.00$ per item. This guarantees that an order will be processed through NTIS within 24 hours of its receipt. These orders receive immediate, individual attention. The items ordered are delivered by first call mail. Call NTIS for information on rush order service for computer products.

For Help in Tracing an Order - Call (703) 487-4650 and request the customer service option.

\section{ADDITIONAL HYDROPOWER EVALUATION SOFTWARE INFORMATION}

Additional information concerning the Hydropower Evaluation Software can be obtained by contacting Ben Rinehart or Jim Francfort at the addresses provided below. Copies of the software and the User's Manual may also be obtained from these individuals.

\author{
Ben Rinehart \\ Idaho National Engineering Laboratory \\ P.O. Box 1625 \\ Idaho Falls, ID 83415-3830 \\ (208) 526-1002 \\ Jim Francfort \\ Idaho National Engineering Laboratory \\ P.O. Box 1625 \\ Idaho Falls, ID 83415-3875 \\ (208) 526-6787
}

Information concerning the state of North Dakota involvement with the resource assessment or about the identified sites may be obtained by contacting:

Kim Christianson

Office of Intergovernmental Assistance

14th Floor - State Capital

600 East Boulevard Avenue

Bismarck, North Dakota 83415-3526

(701) 224-2094

\section{REFERENCES}

Francfort, J. E., S. D. Matthews, and B. N. Rinehart, 1991, Hydropow'er Evaluation Software User's Manual, DOE/ID-10338, Idaho National Engineering Laboratory, Idaho Falls, Idaho.

Francfort, J. E., K. M. Moore, and B. N. Rinehart, 1993, Uniform Criteria for U.S. Hydropower Resource Assessment, Hydropow'er Evaluation Software Status Report, DOE/ID-10430, Idaho National Engineering Laboratory, Idaho Falls, Idaho. 


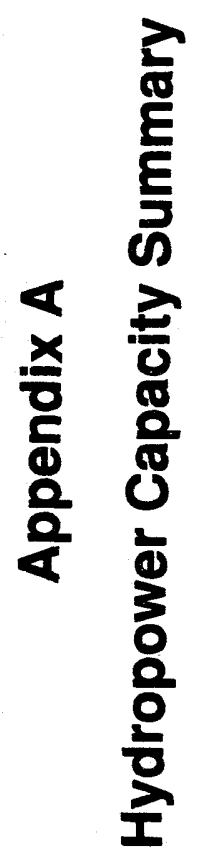




\begin{tabular}{|c|c|c|c|c|}
\hline state & $\begin{array}{l}\text { D R O P O } \\
\text { Category }\end{array}$ & $\begin{array}{l}\text { R C A } \\
\text { Number of } \\
\text { Projects }\end{array}$ & $\begin{array}{l}\text { T Y S U } \\
\text { Name Plate } \\
\text { Capacity (KW) }\end{array}$ & $\begin{array}{l}\text { 10/05/93 } \\
\text { R Y } \\
\text { HES Adjusted } \\
\text { Capacity (KW) }\end{array}$ \\
\hline \multirow[t]{4}{*}{ ND } & With Power & 2 & 86108.50 & 43054.25 \\
\hline & พ/O Power & 10 & 13431.55 & 6718.34 \\
\hline & Undeveloped & 2 & 39 & 35.10 \\
\hline & STATE TOTAL & 14 & 99579.05 & 49807.69 \\
\hline \multirow[t]{3}{*}{ TOTALS } & with Power & 2 & 86108.50 & 43054.25 \\
\hline & w/O Power & 10 & 13431.55 & 6718.34 \\
\hline & Undeveloped & 2 & 39 & 35.10 \\
\hline \multicolumn{2}{|c|}{ GRAND TOTAL } & 14 & 99579.05 & 49807.69 \\
\hline
\end{tabular}


Appendix B

Hydropower Resource Assessment by River Basin 


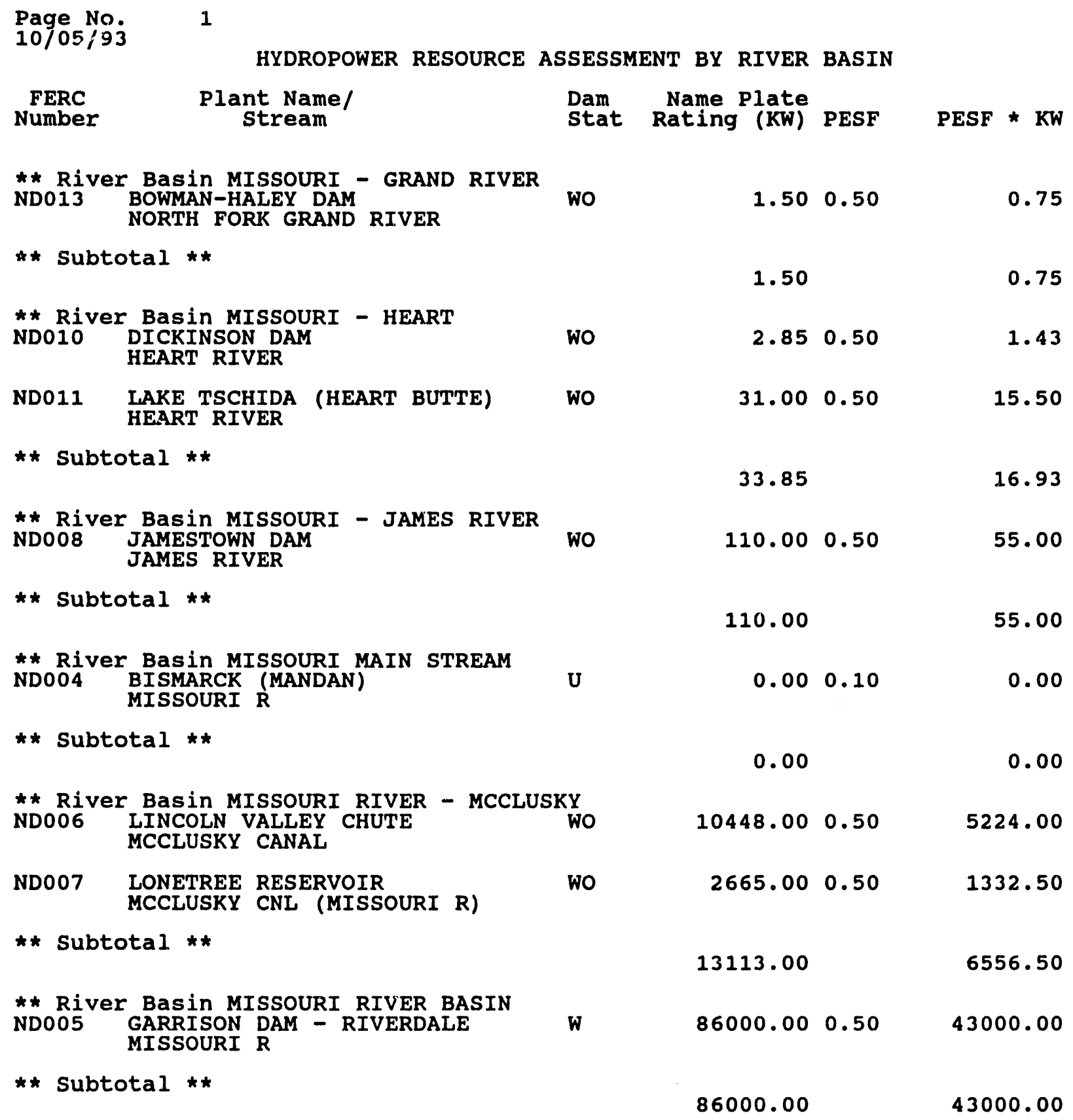




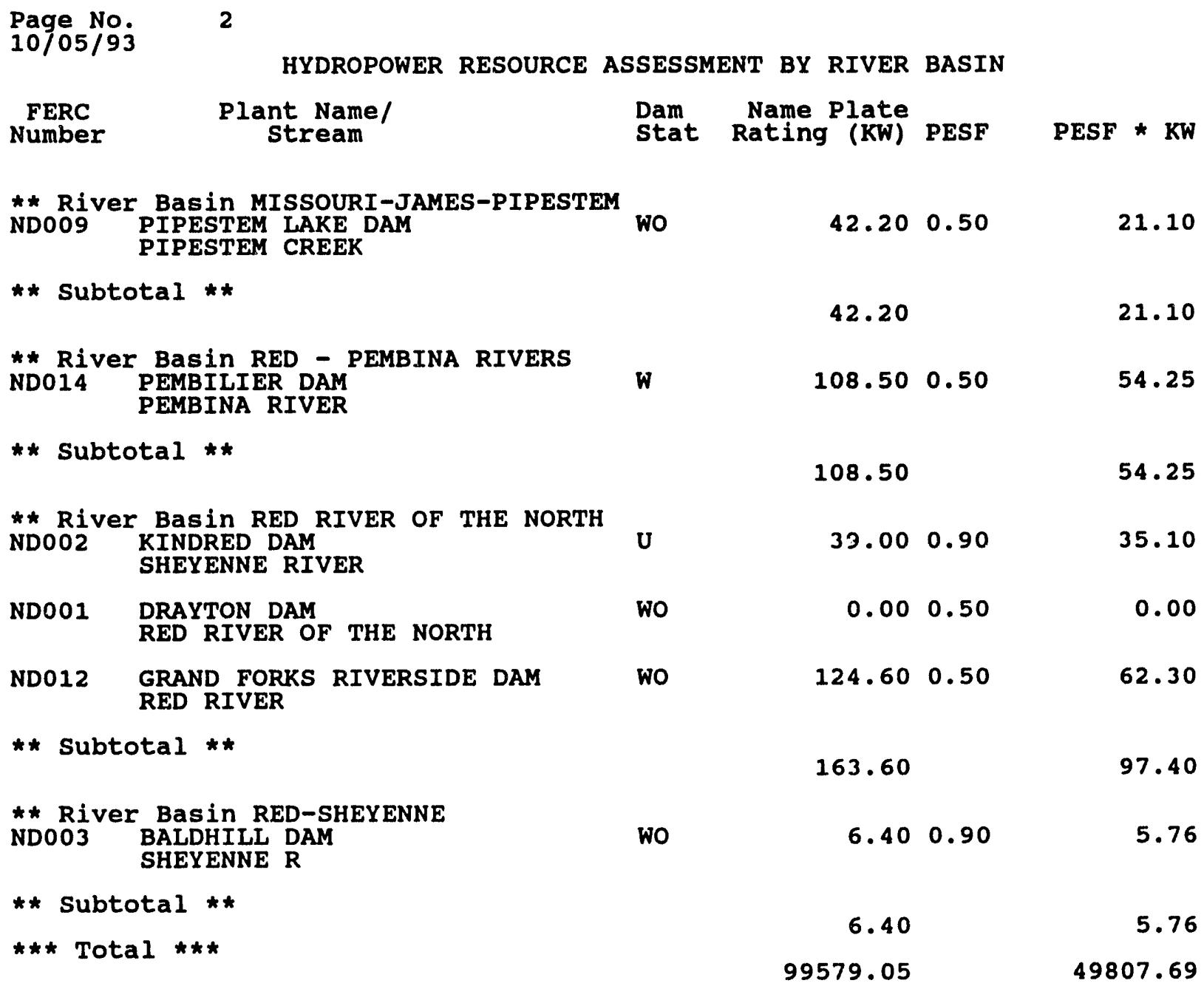




\section{Appendix C \\ North Dakota (ND) Sites}

C-1 


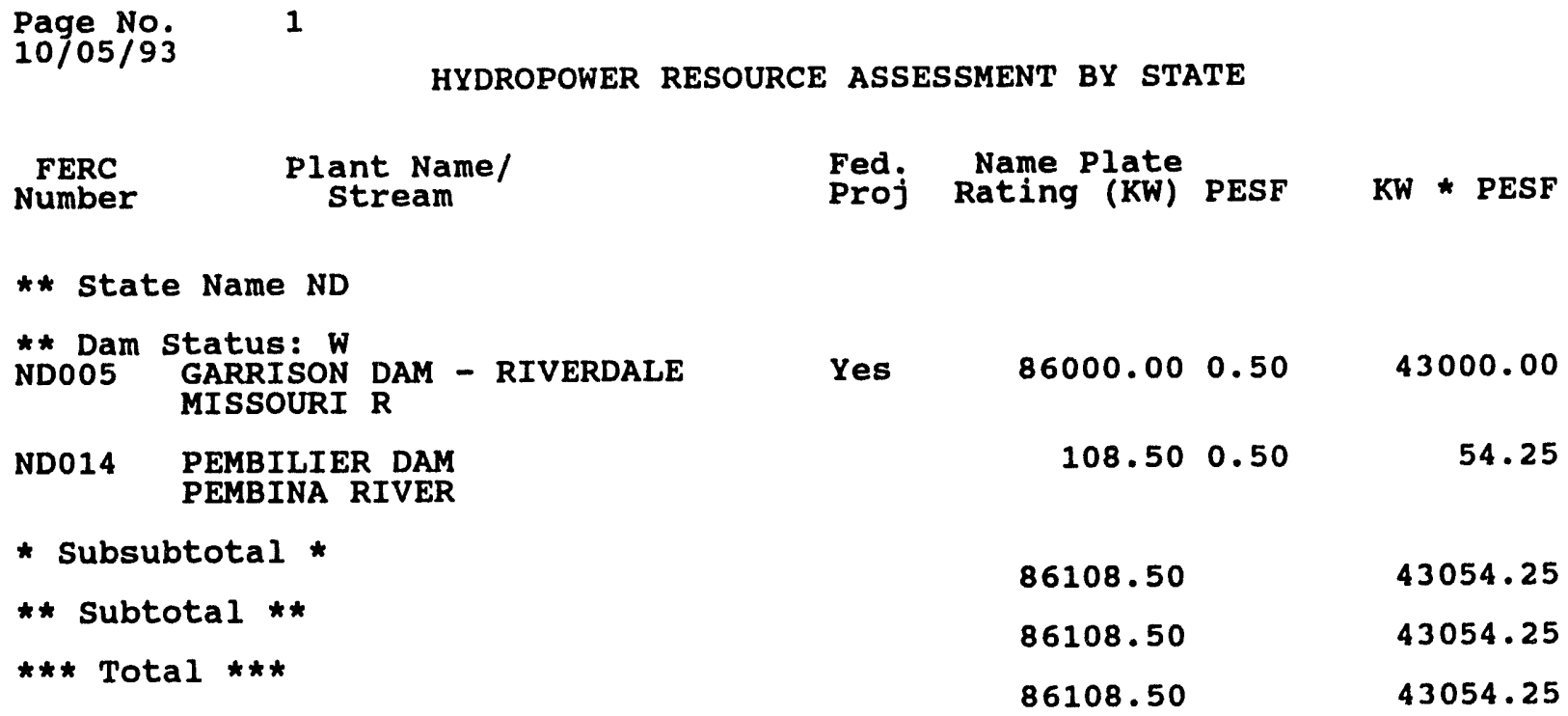


Page No.
$10 / 05 / 93$

FERC

Number

$\underset{\text { Stream }}{\text { Name/ }}$

HYDROPOWER RESOURCE ASSESSMENT BY STATE

* State Name ND

* Dam status: wo

ND003 BALDHILL DAM

SHEYENNE R

ND006 LINCOLN VALLEY CHUTE MCCLUSKY CANAL

ND007 LONETREE RESERVOIR MCCLUSKY CNL (MISSOURI R)

ND008

JAMESTOWN DAM JAMES RIVER

ND009

PIPESTEM LAKE DAM PIPESTEM CREEK

ND010 DICKINSON DAM HEART RIVER

ND011 LAKE TSCHIDA (HEART BUTTE) HEART RIVER

ND013

BOWMAN-HALEY DAM

NORTH FORK GRAND RIVER

ND001 DRAYTON DAM

RED RIVER OF THE NORTH

ND012 GRAND FORKS RIVERSIDE DAM RED RIVER

* Subsubtotal *

* * Subtotal **

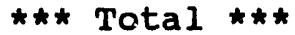

Fed. Name Plate
Proj Rating (KW) PESF KW * PESF

Yes

$6.40 \quad 0.90$

5.76

Yes

$10448.00 \quad 0.50$

5224.00

Yes

2665.000 .50

1332.50

Yes

$110.00 \quad 0.50$

55.00

Yes

42.200 .50

21.10

Yes

2.850 .50

1.43

Yes

$31.00 \quad 0.50$

15.50

Yes

$1.50 \quad 0.50$

0.75

$0.00 \quad 0.50$

0.00

$124.60 \quad 0.50$

62.30

13431.55

6718.34

13431.55

6718.34

13431.55 
Page No.
$10 / 05 / 93$

1

HYDROPOWER RESOURCE ASSESSMENT BY STATE

FERC

Number

Plant Name/

stream

Fed. Name Plate

Proj Rating (KW) PESF

KW * PESF

** State Name ND

** Dam Status: U

NDO04 BISMARCK (MANDAN)

$0.00 \quad 0.10$

0.00

ND002

KINDRED DAM

SHEYENNE RIVER

$39.00 \quad 0.90$

35.10

* Subsubtotal *

** Subtotal **

39.00

35.10

$\star \star \star$ Total $\star * *$

39.00

35.10

39.00

35.10 


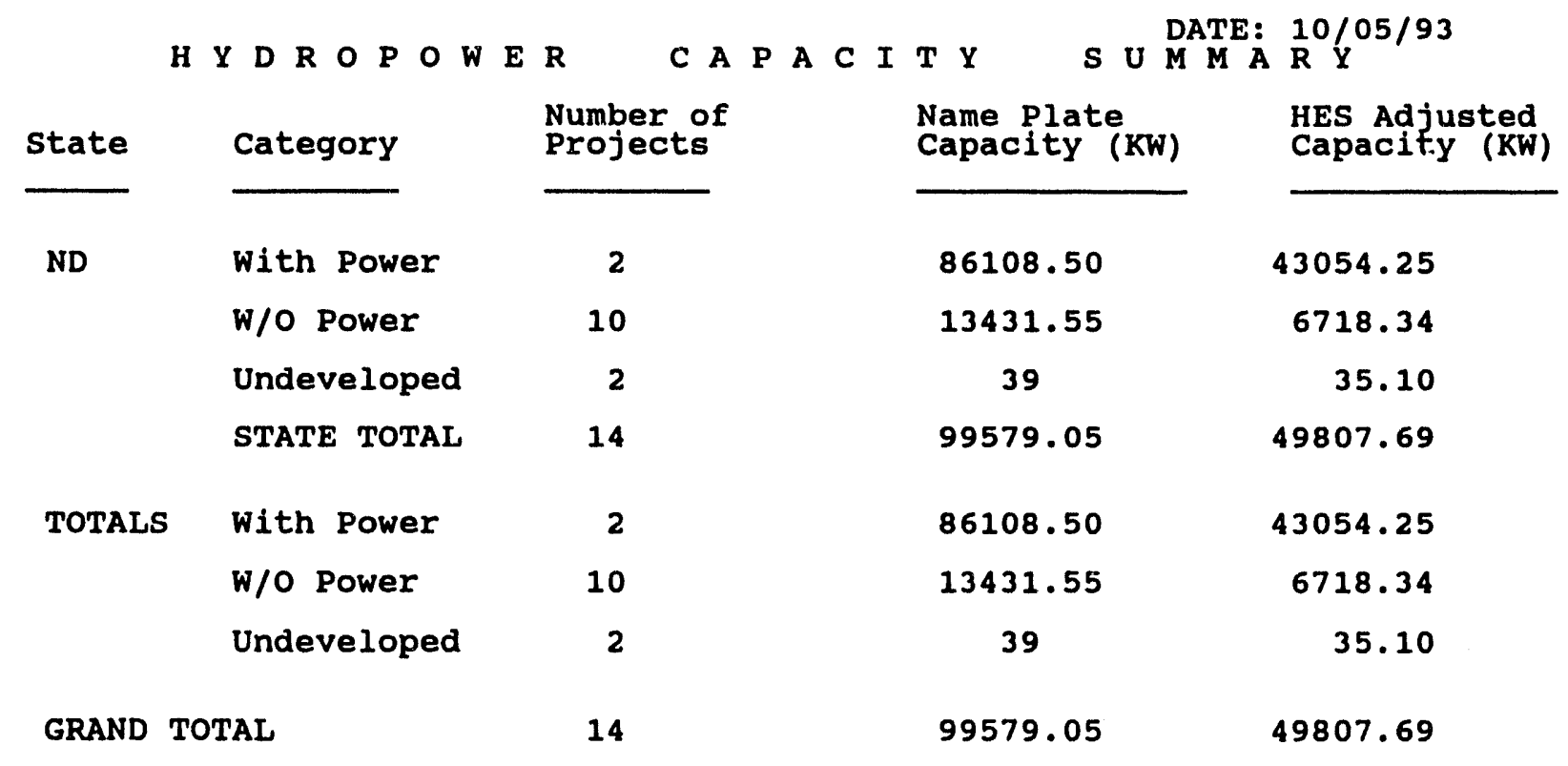




\section{Appendix D \\ Resource Database Listing}

D-1 
RE S O U R C E D A T A B A S E L I S T I N G

DATE: $10 / 05 / 93$

PAGE NO: 1

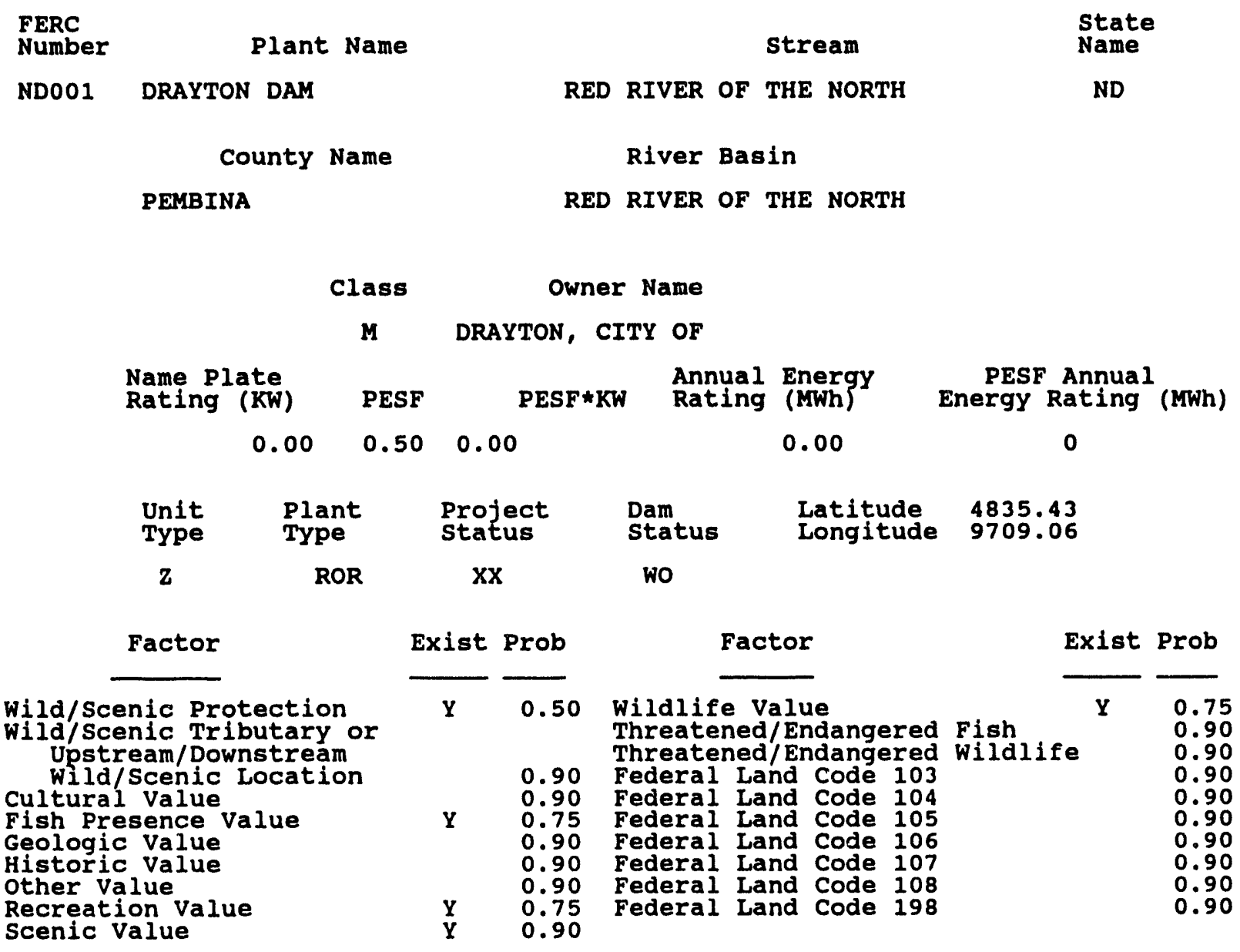


RE S O U R C E D A T A B A S E I I S T I N G

DATE: $10 / 05 / 93$

PAGE NO: 2

FERC

Number

Plant Name

stream

state

NDOO2 KINDRED DAM

SHEYENNE RIVER

Name

County Name

River Basin

RANSOM

RED RIVER OF THE NORTH

Class Owner Name

M RANSOM COUNTY WRD

\begin{tabular}{|c|c|c|c|c|c|c|}
\hline \multicolumn{2}{|c|}{$\begin{array}{l}\text { Name plate } \\
\text { Rating (KW) }\end{array}$} & PESF & PESF $\star K W$ & \multicolumn{2}{|c|}{$\begin{array}{l}\text { Annual Energy } \\
\text { Rating (MWh) }\end{array}$} & $\begin{array}{l}\text { PESF Annual } \\
\text { Energy Rating (MWh) }\end{array}$ \\
\hline & 39.00 & 0.90 & 35.10 & \multicolumn{2}{|c|}{170.90} & 153.81 \\
\hline $\begin{array}{l}\text { Unit } \\
\text { Type }\end{array}$ & \multicolumn{2}{|c|}{$\begin{array}{l}\text { Plant } \\
\text { Type }\end{array}$} & $\begin{array}{l}\text { Project } \\
\text { status }\end{array}$ & $\begin{array}{l}\text { Dam } \\
\text { status }\end{array}$ & \multirow[t]{2}{*}{$\begin{array}{l}\text { Latitude } \\
\text { Longitude }\end{array}$} & \multirow[t]{2}{*}{$\begin{array}{l}4632.00 \\
9818.00\end{array}$} \\
\hline $\mathbf{z}$ & \multicolumn{2}{|c|}{ RES } & zZ & $\mathbf{U}$ & & \\
\hline
\end{tabular}

Factor

wild/Scenic Protection

wild/Scenic iributary or

Upstream/Downstream

wild/Scenic Location

Cultural Value

Fish Presence Value

Geologic Value

Historic Value

other Value

Recreation value

Scenic Value
Exist Prob

Factor

Exist Prob

0.90

0.90

0.90

0.90

0.90

0.90

0.90

0.90

0.90

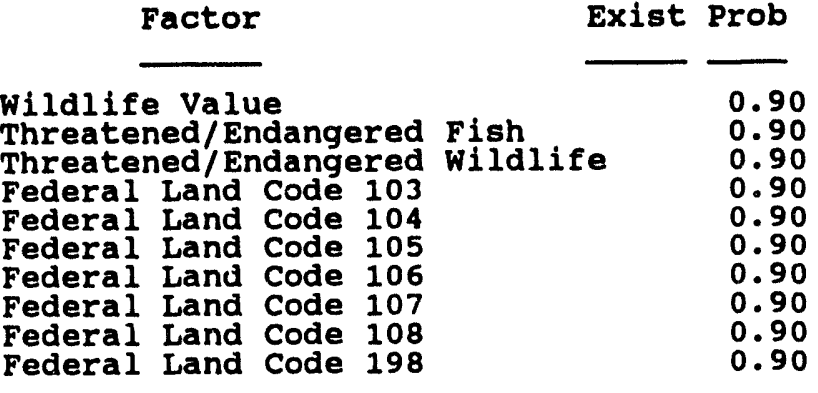

ND 

RE S O U R C E
D A T A B A E
I I $S$ T I N G

DATE: $10 / 05 / 93$

PAGE NO: 3

FERC

Number

Plant Name

stream

state

NDO03

BALDHILL DAM

SHEYENNE $\mathbf{R}$

ND

County Name

BARNES

River Basin

RED-SHEYENNE

$\begin{array}{cc}\text { Class } & \text { Owner Name } \\ \text { F } & \text { CORPS OF ENGINEERS }\end{array}$

\begin{tabular}{|c|c|c|c|c|}
\hline $\begin{array}{l}\text { Name plate } \\
\text { Rating (KW) }\end{array}$ & PESF & PESF $\star K W$ & $\begin{array}{ll}\text { Annual Energy } \\
\text { Rating (MWh) }\end{array}$ & $\begin{array}{l}\text { PESF Annual } \\
\text { Energy Rating (MWh) }\end{array}$ \\
\hline 6.40 & 0.90 & 5.76 & 34.60 & 31.14 \\
\hline
\end{tabular}

$\begin{array}{llll}\text { Unit Plant Project Dam } & \text { Latitude } & \mathbf{4 7 0 2 . 1 2}\end{array}$

Type Type Status status Longitude 9804.48

RES

$\mathrm{XX}$

พอ

Factor

Exist Prob

Factor

Exist Prob

Wild/Scenic Protection

wild/Scenic Tributary or

Upstream/Downstream

wild/Scenic Location

Cultural value

Fish Presence Value

Geologic Value

Historic Value

other Value

Recreation Value

Scenic Value

-1.90
0.90
0.90
0.90
0.90
0.90
0.90
0.90
0.90

Wildlife Value

Threatened/Endangered Fish Threatened/Endangered Wildlife

0.90

0.90

0.90

0.90

Land Code 103

Federal Land Code 104

Federal Land Code 105

Federal Land Code 106

Federal Land Code 107

Federal Land Code 108

Federal Land Code 198

0.90

0.90

0.90

0.90

0.90 
RE S O UR C E D A T A B S E L I S T I N G

DATE: $10 / 05 / 93$

PAGE NO: 4

FERC

Number

Plant Name

stream

State

Name

ND004 BISMARCK (MANDAN)

MISSOURI R

ND

County Name

River Basin

BURLEIGH

MISSOURI MAIN STREAM

Class Owner Name

0

\begin{tabular}{|c|c|c|c|c|c|}
\hline $\begin{array}{l}\text { Name Plate } \\
\text { Rating (KW) }\end{array}$ & PESF & PESF $\star K W$ & $\begin{array}{l}\text { Annual } \\
\text { Rating }\end{array}$ & $\begin{array}{l}\text { Energy } \\
\text { (MWh) }\end{array}$ & $\begin{array}{l}\text { PESF Annual } \\
\text { Energy Rating (MWh) }\end{array}$ \\
\hline 0.00 & 0.10 & & & 0.00 & 0 \\
\hline
\end{tabular}

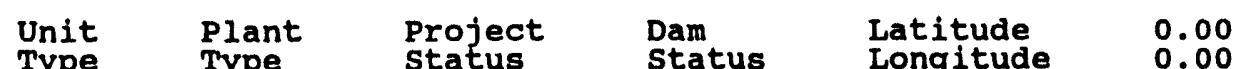

Type Type status

Z

ROR

ZZ

$\mathrm{U}$

\begin{tabular}{|c|c|c|c|c|c|c|}
\hline Factor & Exist & Prob & & Factor & Exist & Prob \\
\hline $\begin{array}{l}\text { Wild/Scenic Protection } \\
\text { wild/Scenic Tributary or } \\
\text { Upstream/Downstream } \\
\text { Wild/Scenic Location } \\
\text { Cultural Value } \\
\text { Fish Presence Value } \\
\text { Geologic Value } \\
\text { Historic Value } \\
\text { other Value } \\
\text { Recreation Value } \\
\text { Scenic Value }\end{array}$ & $\begin{array}{l}\mathbf{Y} \\
\mathbf{Y} \\
\mathbf{Y} \\
\mathbf{Y} \\
\mathbf{Y}\end{array}$ & $\begin{array}{l}0.90 \\
0.90 \\
0.50 \\
0.25 \\
0.90 \\
0.50 \\
0.90 \\
0.25 \\
0.50\end{array}$ & $\begin{array}{l}\text { Wildlife } \\
\text { Threaten } \\
\text { Threaten } \\
\text { Federal } \\
\text { Federal } \\
\text { Federal } \\
\text { Federal } \\
\text { Federal } \\
\text { Federal } \\
\text { Federal }\end{array}$ & $\begin{array}{l}\text { V Value } \\
\text { ned/Endangered } \\
\text { ned/Endangered } \\
\text { Land Code } 103 \\
\text { Land Code } 104 \\
\text { Land Code } 105 \\
\text { Land Code } 106 \\
\text { Land Code } 107 \\
\text { Land Code } 108 \\
\text { Land Code } 198\end{array}$ & $\begin{array}{l}\text { Fish } \\
\text { Wildife }\end{array}$ & $\begin{array}{l}0.25 \\
0.90 \\
0.90 \\
0.90 \\
0.90 \\
0.90 \\
0.90 \\
0.90 \\
0.90 \\
0.90\end{array}$ \\
\hline
\end{tabular}


RE S O U R C E D A T A B A S E IA I S T I N G

DATE: $10 / 05 / 93$

PAGE NO: 5

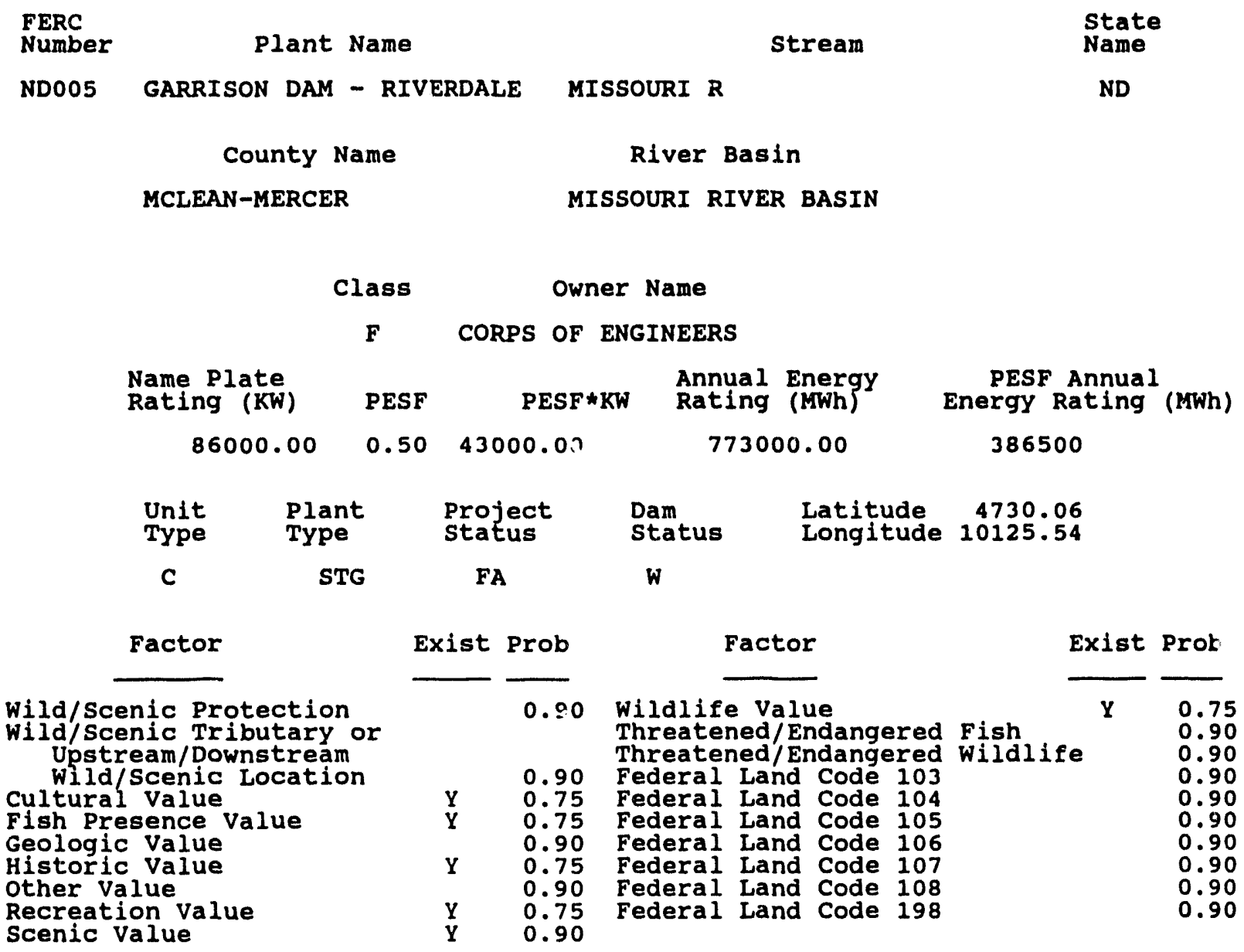


RE S O U R C E D A T A B A S E L I S T I N G

DATE: $10 / 05 / 93$

PAGE NO: 6

FERC

Number

Plant Name

stream

State

ND006

IINCOLN VALLEY CHUTE

County Name

MCCLUSKY CANAL

River Basin

SHERIDAN

MISSOURI RIVER - MCCLUSKY

$\begin{array}{cc}\text { Class } & \text { Owner Name } \\ \text { F } & \text { BUREAU OF RECLAMATION }\end{array}$

Name Plate
Rating $(K W)$

PESF $\quad$ PESF $* K W \quad \begin{aligned} & \text { Annual Energy } \\ & \text { Rating (MWh) }\end{aligned}$

PESF Annual

$$
10448.00
$$

$0.50 \quad 5224.00$

59088.00 Energy Rating (MWh)

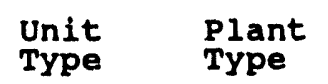

Z

CMB

Factor

wild/Scenic Protection

wild/Scenic Tributary or

Upstream/Downstream

wild/scenic Location

Cultural value

Fish Presence Value

Geologic value

Historic Value

other value

Recreation Value

Scenic Value

\begin{tabular}{l}
$\begin{array}{l}\text { Project } \\
\text { Ptatus }\end{array}$ \\
Zz \\
xist \\
Prob \\
\hline
\end{tabular}

29544

4742.00

10020.15
ND

Name 

R E S O U R C E
D A T A B A S E
L I S T I N G

DATE: $10 / 05 / 93$

FERC

Number

Plant Name

ND007

LONETREE RESERVOIR

County Name

PAGE NO: 7

County Name
SHERIDAN

$\begin{array}{cc}\text { Stream } & \begin{array}{c}\text { State } \\ \text { Name }\end{array} \\ \text { MCCLUSKY CNL (MISSOURI R) } & \text { ND } \\ \text { River Basin } & \\ \text { MISSOURI RIVER - MCCLUSKY }\end{array}$

class Owner Name

F BUREAU OF RECLAMATION

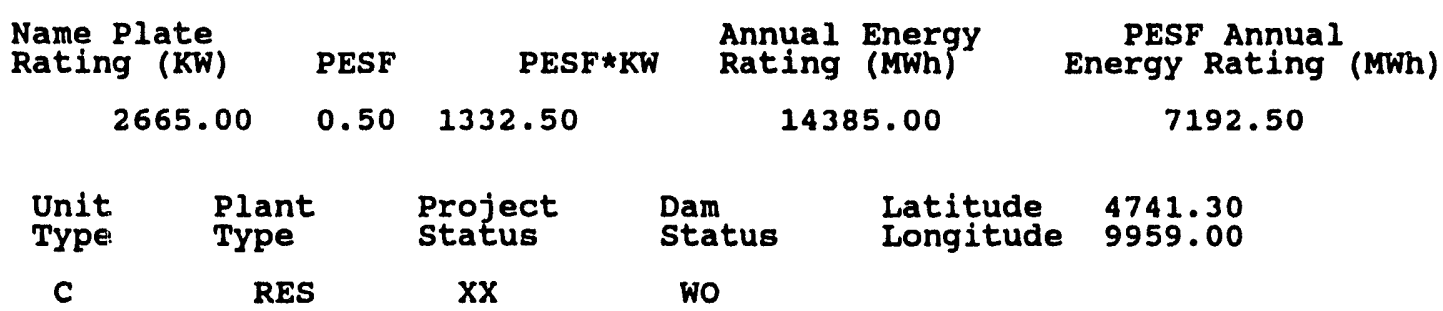

Factor

Exist Prob

Factor

Exist Prob

Wild/Scenic Protection

Wild/Scenic Tributary or

Upstream/Downstream

wild/Scenic Location

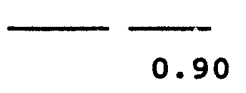

0.90

fe Value

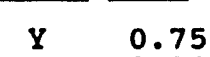

Wildilfe Value

Thedeater Wilde

0.90

0.90

Cultural Value

Fish Presence Value

Geologic Value

Federal Land Code 104

0.75 Federal Land Code 105

0.90 Federal Land Code 106

0.90

Historic Value

other Value

Recreation Value

Y $\quad 0.75$ Federal Land Code 107

0.90

0.90

$\begin{array}{lll} & 0.90 & \text { Federal Land Code } 108 \\ Y & 0.75 & \text { Federal Land Code } 198\end{array}$

0.90

0.90

0.90

scenic Value

0.90

0.90 
RES O UR C E DA TABASE L I S T I N G

DATE: $10 / 05 / 93$

PAGE NO: 8

FERC

Number

ND008
Plant Name

JAMESTOWN DAM

County Name

STUTSMAN

\author{
stream $\quad \begin{gathered}\text { State } \\ \text { Name }\end{gathered}$ \\ ND \\ JAMES RIVER \\ River Basin \\ MISSOURI - JAMES RIVER
}

Class Owner Name

F BUREAU OF RECLAMATION

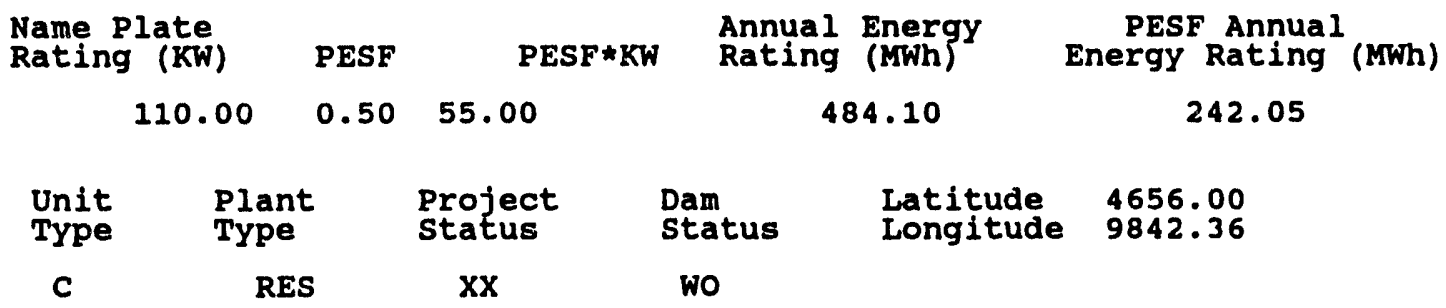

Factor

Wild/Scenic Protection wild/Scenic Tributary or Upstream/Downstream wild/scenic Location Cultural value Fish Presence Value Geologic Value Historic Value other Value Recreation Value Scenic Value
Exist Prob

Factor

0.90

Wildlife Value Threatened/Endangered Fish Threatened/Endangered Wildilfe Exist Prob

$\begin{array}{lll}0.90 & \text { Federal Land Code } 103 \\ 0.90 & \text { Federal Land Code } 104\end{array}$

$\checkmark \quad 0.75$ Federal Land Code 105

0.90 Federal Land Code 106

0.90 Federal Land Code 107

0.90 Federal Land Code 108

0.90 Federal Land Code 198

0.90

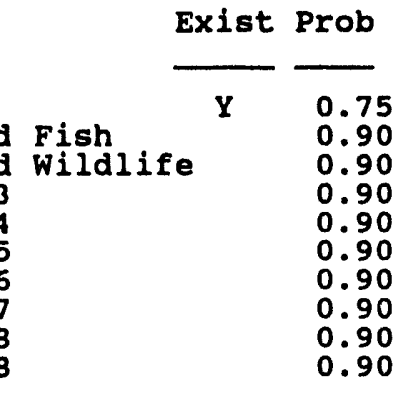


RES O UR C E D A T A B A S E L I S T I N G

DATE: $10 / 05 / 93$

PAGE NO: 9

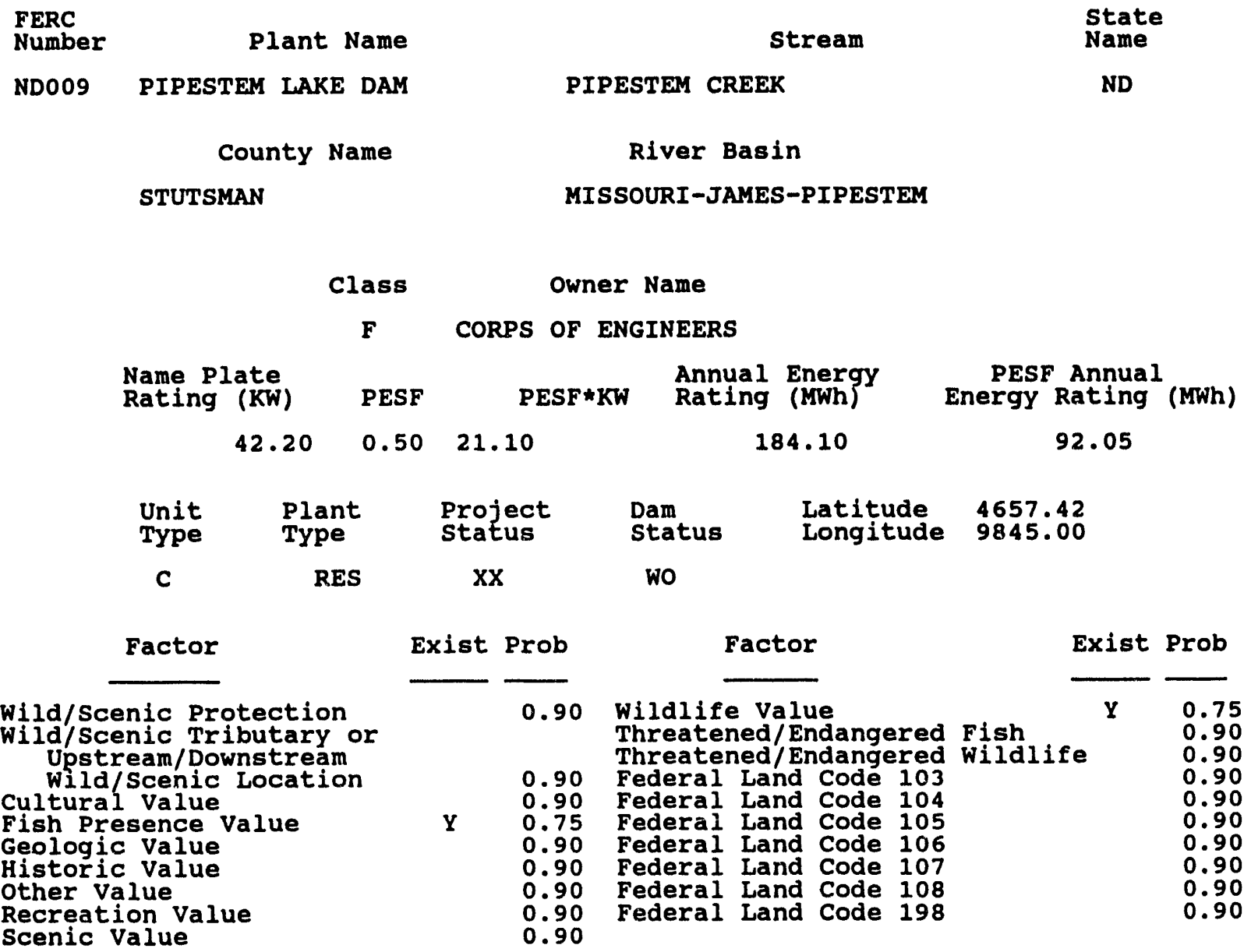



R E S O U R C E
D A T A B A $\mathbf{E}$
L I $S$ T I N G

DATE: $10 / 05 / 93$

PAGE NO: 10

FERC

Number

Plant Name

stream

State

ND010

DICKINSON DAM

HEART RIVER

ND

County Name

River Basin

STARK

MISSOURI - HEART

class Owner Name

F BUREAU OF RECLAMATION

\begin{tabular}{|c|c|c|c|c|c|}
\hline $\begin{array}{l}\text { Name Plate } \\
\text { Rating (KW) }\end{array}$ & PESF & PESF $\star K W$ & $\begin{array}{l}\text { Annual } \\
\text { Rating }\end{array}$ & $\begin{array}{l}\text { Energy } \\
\text { (MWh) }\end{array}$ & $\begin{array}{l}\text { PESF Annual } \\
\text { Energy Rating (MWh) }\end{array}$ \\
\hline 2.85 & 0.50 & 1.43 & & 9.98 & 4.99 \\
\hline
\end{tabular}

$\begin{array}{lllll}\text { Unit } & \text { Plant } & \text { Project } & \text { Dam } & \text { Latitude } \\ \text { Type } & \text { Type } & \text { Status } & \text { Status } & \text { Longitude } 10249.32\end{array}$

C

RES

$\mathbf{X X}$

Wo

Factor

Exist Prob

Factor

Exist Prob

Wild/Scenic Protection

wild/Scenic Tributary or Upstream/Downstream wild/Scenic Location Cultural value

Fish Presence Value

Geologic Value

Historic Value

other Value

Recreation Value

Scenic Value

\begin{tabular}{|c|c|}
\hline & 0.90 \\
\hline $\mathbf{Y}$ & $\begin{array}{l}0.90 \\
0.90 \\
0.75 \\
0.90 \\
0.90 \\
0.90 \\
0.90 \\
0.90\end{array}$ \\
\hline
\end{tabular}

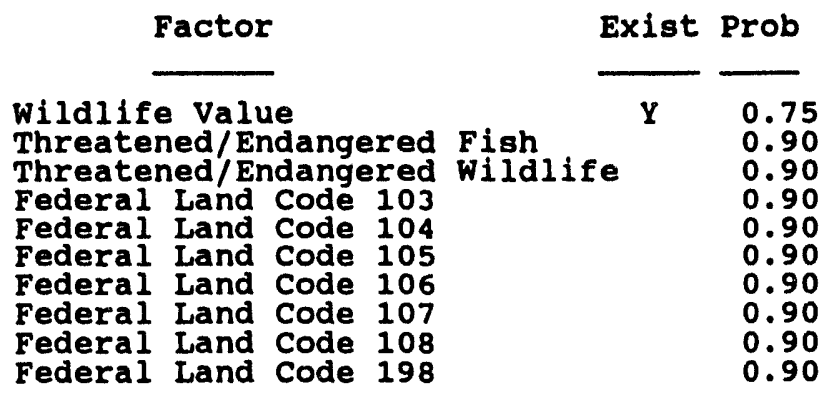


RE S O U R C E D A T A B A S E L I S T I N G

DATE: $10 / 05 / 93$

PAGE NO: 11

FERC

Number

Plant Name

stream

state

ND011

LAKE TSCHIDA (HEART BUTTE) HEART RIVER

ND

County Name

River Basin

GRANT

MISSOURI - HEART

Class Owner Name

F BUREAU OF RECLAMATION

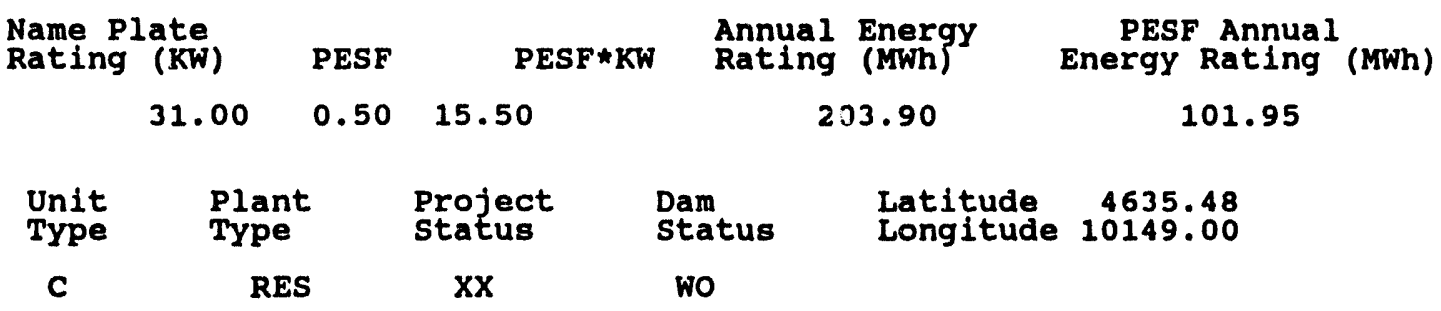

Factor

Exist Prob

Factor

Exist Prob

Wild/Scenic Protection

wild/Scenic Tributary or

Upstream/Downstream

wild/Scenic Location

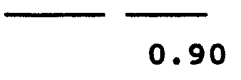

0.90

Wildlife Value

Y

Threatened/Endangered Fish

Threatened/Endangered Wildlife

0.90 Federal Land Code 103

Cultural value

Fish Presence Value

0.90 Federal Land Code 104

0.75 Federal Land Code 105

0.90 Federal Land Code 106

0.90 Federal Land Code 107

0.90 Federal Land Code 108

Historic value

other Value

0.90

Federal Land Code 198

0.90

0.90

0.90

0.90

0.90

scenic Value 
RE S O U R C E DA TA B A S E L I S T I N G

DATE: $10 / 05 / 93$

PAGE NO: 12

FERC

Number

Plant Name

stream

State

Name

ND012

GRAND FORKS RIVERSIDE DAM RED RIVER

ND

County Name

GRAND FORKS

River Basin

RED RIVER OF THE NORTH

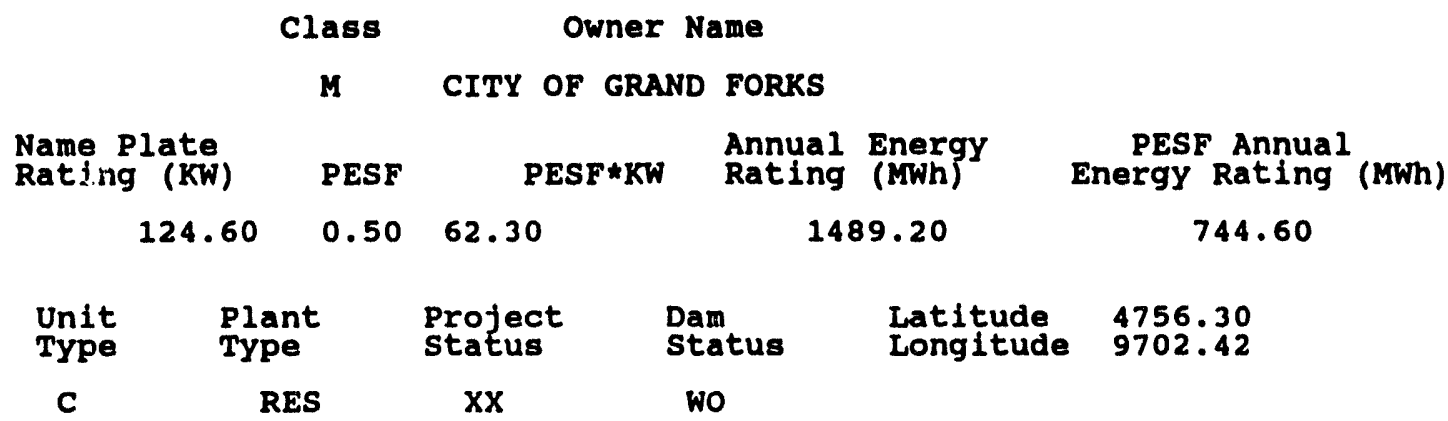

Factor

Exist Prob

Factor

Exist Prob

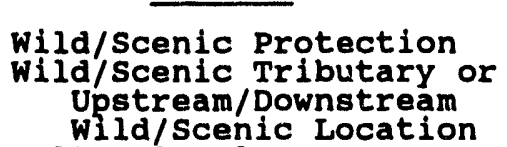
wild/Scenic Location Cultural Value Fish Presence Value Geologic Value Historic Value other Value Recreation Value scenic Value

\begin{tabular}{cc} 
& \\
\hline & 0.90 \\
& \\
& 0.90 \\
$Y \quad 0.90$ \\
0.75 \\
0.90 \\
0.90 \\
0.90 \\
0.90 \\
0.90
\end{tabular}

-0.75
0.90
0.90
0.90
0.90
0.90
0.90
0.90
0.90
0.90


R E S O U R C E

DATE: $10 / 05 / 93$

FERC

Number

ND013
Plant Name

BOWMAN-HALEY DAM

County Name

BOWMAN
D A T A B A S E

L I $\mathbf{S}$ T I $\mathbf{N}$ G

PAGE NO: 13

stream

NORTH FORK GRAND RIVER

River Basin

MISSOURI - GRAND RIVER

\begin{tabular}{|c|c|c|c|c|c|}
\hline \multirow[b]{3}{*}{$\begin{array}{l}\text { Name Plate } \\
\text { Rating (KW) }\end{array}$} & 1 ass & \multicolumn{2}{|c|}{ Owner Name } & & \\
\hline & $\boldsymbol{F}$ & CORPS OF EN & NEERS & & \\
\hline & PESF & PESF $\star K W$ & $\begin{array}{l}\text { Annual } \\
\text { Rating }\end{array}$ & $\begin{array}{l}\text { Energy } \\
\text { (MWh) }\end{array}$ & $\begin{array}{l}\text { PESF Annual } \\
\text { Energy Rating (MWh) }\end{array}$ \\
\hline 1.50 & 0.50 & 0.75 & & 6.90 & 3.45 \\
\hline
\end{tabular}

Unit Plant Project Dam Latitude $\mathbf{4 3 5 8 . 5 4}$

Type Type status status Longitude 10314.48

C

RES

$\mathbf{X X}$

พอ

Factor

Exist Prob

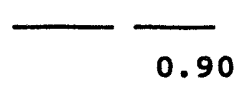

0.90

ild/Scenic Protection

Wild/Scenic Tributary or Upstream/Downstream wild/Scenic Location Cultural value

Fish Presence Value

Geologic Value

Historic Value

other Value

Recreation Value

Scenic Value

0.75

0.90

0.90

0.90

0.90

0.90

\begin{tabular}{|c|c|c|c|}
\hline & Factor & Exist & Prob \\
\hline $\begin{array}{l}\text { Wildlife } \\
\text { Threaten } \\
\text { Threaten } \\
\text { Federal } \\
\text { Federal } \\
\text { Federal } \\
\text { Federal } \\
\text { Federal } \\
\text { Federal } \\
\text { Federal }\end{array}$ & $\begin{array}{l}\text { e Value } \\
\text { ned/Endangered } \\
\text { ned/Endangered } \\
\text { Land Code } 103 \\
\text { Land Code } 104 \\
\text { Land Code } 105 \\
\text { Land Code } 106 \\
\text { Land Code } 107 \\
\text { Land Code } 108 \\
\text { Land Code } 198\end{array}$ & $\begin{array}{l}\text { Fish } \\
\text { Wildife }\end{array}$ & $\begin{array}{l}0.75 \\
0.90 \\
0.90 \\
0.90 \\
0.90 \\
0.90 \\
0.90 \\
0.90 \\
0.90 \\
0.90\end{array}$ \\
\hline
\end{tabular}


R E S O U R C E D A T A B A S E L I S T I N G

DATE: $10 / 05 / 93$

PAGE NO: 14

FERC

Number

ND014
Plant Name

PEMBILIER DAM

County Name

PEMBINA stream

PEMBINA RIVER

River Basin

RED - PEMBINA RIVERS

\section{Class Owner Name}

$M$ PEMBINA COUNTY WRD

\begin{tabular}{|c|c|c|c|c|}
\hline $\begin{array}{l}\text { Name Plate } \\
\text { Rating (KW) }\end{array}$ & PESF & $P E S F \star K W$ & $\begin{array}{l}\text { Annual Energy } \\
\text { Rating (MWh) }\end{array}$ & $\begin{array}{l}\text { PESF Annual } \\
\text { Energy Rating (MWh) }\end{array}$ \\
\hline 108.50 & 0.50 & 54.25 & 477.20 & 238.60 \\
\hline
\end{tabular}

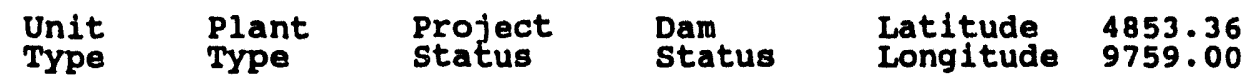

C

RES

Factor

Wild/Scenic Protection

Wild/Scenic Tributary or Upstream/Downstream wild/Scenic Location Cultural value Fish Presence Value Geologic Value Historic Value other Value Recreation Value scenic value
Exist Prob

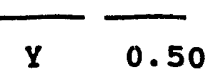

$Y$
$Y$
$Y$
$Y$
$Y$
$Y$
$Y$
$Y$ $\mathbf{x x} \boldsymbol{W}$ state

Name

ND

\begin{tabular}{|c|c|c|c|c|}
\hline & Factor & & $x i s t$ & Prob \\
\hline $\begin{array}{l}\text { Wildlife } \\
\text { Threaten } \\
\text { Threaten } \\
\text { Federal } \\
\text { Federal } \\
\text { Federal } \\
\text { Federal } \\
\text { Federal } \\
\text { Federal } \\
\text { Federal }\end{array}$ & $\begin{array}{l}\text { Value } \\
\text { ned/Endangered } \\
\text { ned/Endangered } \\
\text { Land Code } 103 \\
\text { Land Code } 104 \\
\text { Land Code } 105 \\
\text { Land Code } 106 \\
\text { Land Code } 107 \\
\text { Land Code } 108 \\
\text { Land Code } 198\end{array}$ & $\begin{array}{l}\text { Fish } \\
\text { Wildiffe }\end{array}$ & $Y$ & $\begin{array}{l}0.75 \\
0.90 \\
0.90 \\
0.90 \\
0.90 \\
0.90 \\
0.90 \\
0.90 \\
0.90 \\
0.90\end{array}$ \\
\hline
\end{tabular}



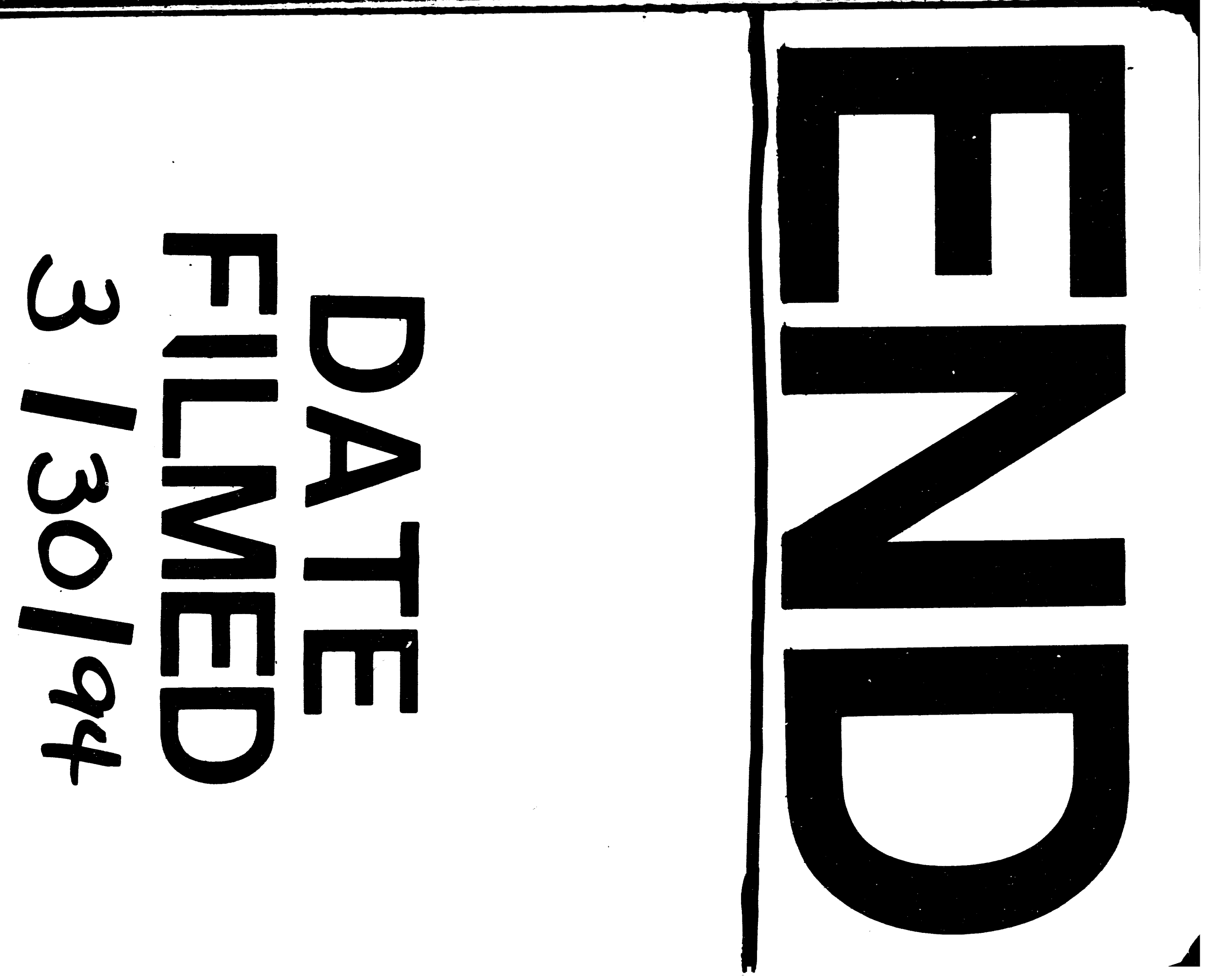
L

$\longrightarrow$

$-$

$\square$ 